Classification

Physics Abstracts

$44.90-87.60$

\title{
La représentation physique des interactions thermiques entre le corps et l'ambiance. Comparaison des modèles élaborés en Europe et aux Etats- Unis
}

\author{
J. J. Roux, P. Depecker et J. M. Pallier
}

Centre de Thermique, INSA, Bât. 307, 20 Avenue A. Einstein, 69621 Villeurbanne Cedex, France

(Reçu le 14 juin 1988, révisé le 18 mai 1989, accepté le 25 septembre 1989)

\begin{abstract}
Résumé. - L'étude présentée porte sur la comparaison des différents modèles de représentation des échanges thermiques entre le corps humain et l'enceinte dans laquelle il se trouve. Il s'agit des modèles danois (Université de Copenhague), américain (Université de Berkeley) et français (CNRS Laboratoire d'Ecothermique de Sophia Antipolis). Dans un premier temps, les auteurs posent le problème de la variété des modèles disponibles, avec les difficultés de choix qu'ils induisent. Puis, on décrit la méthode d'étude, en identifiant les éléments sur lesquels va porter la comparaison : flux échangés, sensation thermique, indice de qualité. Le corps du texte est consacré à la description parallèle de chaque modèle, et aux commentaires des résultats obtenus, sur deux séquences de 48 heures correspondant à des signaux thermiques différents (séquence douce, pendant laquelle la température d'air varie entre 19 et $25^{\circ} \mathrm{C}$ et séquence chaude entre 20 et $40^{\circ} \mathrm{C}$ ). Les auteurs concluent à une divergence inattendue des modèles, en posant le problème prospectif de la représentation thermo-physiologique du phénomène.
\end{abstract}

\begin{abstract}
The aim of the study is the comparison of models describing heat exchanges between human body and his physic environment. Three models are investigated : the Danish model (elaborated by Copenhagen University), the American model (elaborated by Berkeley University), and the French model (elaborated by CNRS). First of all, autors give a scope of the problem, about avalaible diversity models, and so, consequently, choice difficulty. Then, method is described, identifying criterium used for the comparison : heat flux, thermal feeling, quality index. The main part of the text is a parallel description of each model, giving comments of results, about two climatic sequence, the air temperature varying in the range $19-25^{\circ} \mathrm{C}$, the second a hot sequence, with a range of $20-40^{\circ} \mathrm{C}$. Autors conclude on the unexpected diverging results, setting the problem of thermo-physic modelisation of the phenomena in the future.
\end{abstract}

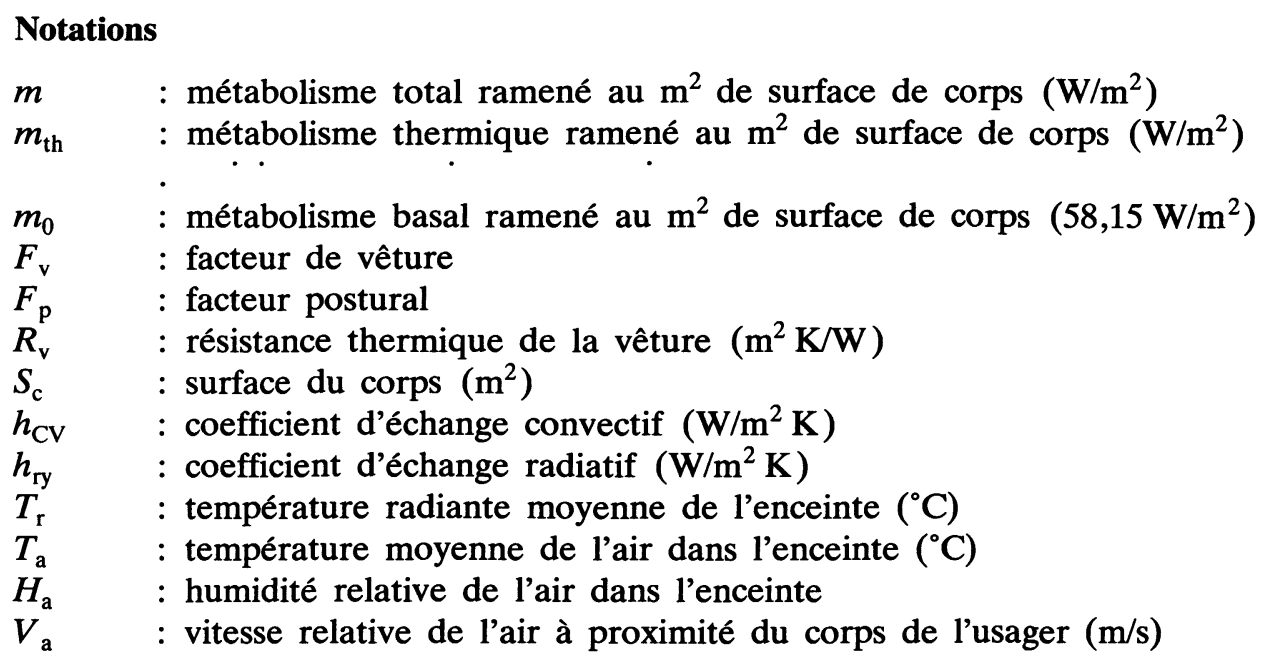


$T_{\mathrm{c}} \quad:$ température cutanée $\left({ }^{\circ} \mathrm{C}\right)$

$T_{\mathrm{d}} \quad:$ température de rosée de l'air dans l'enceinte $\left({ }^{\circ} \mathrm{C}\right)$

$Y \quad:$ vote moyen prévisible $=$ PMV : Scalaire de sensation thermique

$Y_{\text {ry }} \quad:$ coefficient radiatif de $Y$

$Y_{\mathrm{CV}} \quad$ : coefficient convectif de $Y$

$Y_{\mathrm{ev}} \quad$ : coefficient évaporatif de $Y$

$Y_{0} \quad$ : coefficient de base de $Y$

$E_{\mathrm{CV}}(X)$ : densité de flux de chaleur échangé par convection selon le modèle de l'auteur $X\left(\mathrm{~W} / \mathrm{m}^{2}\right)$

$E_{\mathrm{ry}}(X)$ : densité de flux de chaleur échangé par rayonnement selon le modèle de l'auteur $X$ $\left(\mathrm{W} / \mathrm{m}^{2}\right)$

$E_{\mathrm{cr}}(X)$ : chaleur échangée par convection respiratoire par unité de temps, ramenée au $\mathrm{m}^{2}$ de surface de corps, selon le modèle de l'auteur $X\left(\mathrm{~W} / \mathrm{m}^{2}\right)$

$\mathrm{E}_{\mathrm{ps}}(X)$ : chaleur perdue par perspiration par unité de temps, ramenée au $\mathrm{m}^{2}$ de surface de corps, selon le modèle de l'auteur $X\left(\mathrm{~W} / \mathrm{m}^{2}\right)$

$E_{\mathrm{hr}}(X)$ : chaleur perdue par rejet d'humidité respiratoire par unité de temps, ramenée au $\mathrm{m}^{2}$ de surface de corps, selon le modèle de l'auteur $X\left(\mathrm{~W} / \mathrm{m}^{2}\right)$

$E_{\text {sd }}(X)$ : chaleur perdue par sudation par unité de temps, ramenée au $\mathrm{m}^{2}$ de surface de corps, selon le modèle de l'auteur $X\left(\mathrm{~W} / \mathrm{m}^{2}\right)$

$p_{\mathrm{a}} \quad:$ pression atmosphérique de référence $(\mathrm{mmHg})$

$p_{\mathrm{v}}(T) \quad$ : pression partielle de vapeur à la température $T(\mathrm{mmHg})$

$p_{\mathrm{vs}}(T)$ : pression de vapeur saturante à la température $T(\mathrm{mmHg})$

$T^{*} \quad:$ variable température, exprimée en degré Kelvin (K)

$q \quad:$ indice de qualité

$t \quad:$ variable temps

$T_{\mathrm{A}} \quad:$ température résultante dans le modèle de Deval $\left({ }^{\circ} \mathrm{C}\right)$

$w_{\mathrm{a}} \quad$ : humidité absolue de l'air ambiant $(\mathrm{g} / \mathrm{kg})$

$D \quad$ : différentiel thermique $\left(\mathrm{W} / \mathrm{m}^{2}\right)$

$I_{\text {cle }} \quad$ : isolement effectif de la vêture (clo)

$F_{\mathrm{v}, \mathrm{e}} \quad$ : facteur de perméabilité de la vêture

\section{Introduction.}

La représentation physique des interactions thermiques entre le corps et l'ambiance dans laquelle l'usager évolue a fait, depuis plusieurs décennies, l'objet de nombreux travaux essentiellement dirigés vers l'ergonomie. La recherche et l'observation des conditions de confort sont généralement les objectifs principaux de ces études, le champ d'application étant celui des espaces habitables embarqués (aéronautique, astronautique, véhicules au sol), ou celui, très diversifié, des bâtiments d'habitation au sol.

Les modèles élaborés pour représenter ces phénomènes sont, dans la plupart des cas, fondés sur le régime permanent des échanges de chaleur. C'est-àdire que, d'une part, les transferts thermiques au travers de l'enveloppe du corps (le "vêtement ») sont considérés comme peu capacitifs et que, d'autre part, le régime thermique du corps humain lui-même est stabilisé, le recours à l'équation générale de la chaleur devenant inutile. Le but de notre travail est ici de comparer les résultats obtenus, en termes de sensation thermique, à l'aide de 3 modèles très connus, dus aux auteurs suivants :

* modèle de P. O. Fanger (noté 1), de l'université du Danemark ;
* modèle de J. Deval et X. Berger (noté 2), du CNRS français;

* modèle de Max Sherman (noté 3) de l'université de Berkeley (USA).

\section{Objectifs, problématique et méthode d'étude.}

2.0 ObJeCtif DE L'ETUdE. - L'objectif de ce travail est de comparer les différents modèles élaborés en Europe et aux USA permettant d'évaluer la sensation thermique (notée $Y$ dans la suite du texte) ressentie par un individu placé dans une ambiance. Cette sensation thermique, prédite par le modèle, dépend étroitement des échanges thermiques décrits par les lois de transfert corps-ambiance. Comparer les modèles prédictifs de la sensation thermique $Y$ se ramène donc implicitement à l'analyse des écarts sur chaque mode de transfert, c'est-à-dire à la dispersion des valeurs de flux auxquelles conduisent les lois d'échange établies par les différents auteurs (Fanger, Deval, Sherman).

Cette comparaison des modèles de prédiction de la sensation thermique $Y$ est d'une grande importance pour la production des outils de la recherche et de l'ingéniérie: connaissance et prédiction des ambiances pour l'habitat embarqué (aérospaciale, 
aéronautique), prédiction des situations extrêmes (engins de combat, milieu hostile), vérification des conditions de confort dans les bâtiments (espace de production, espace hospitalier, habitat classique...).

Au-delà de la stricte comparaison, cette étude devrait permettre d'indiquer à l'utilisateur d'un modèle, la situation relative de la réponse qu'il donne (c'est-à-dire la sensation thermique) par rapport aux autres.

2.1 Problématique. - La comparaison des différents modèles élaborés par les chercheurs pose naturellement le problème des critères sur lesquels on va mener cette comparaison. Car l'on peut, dans un premier temps, envisager de décomposer ces modèles et de rapprocher les différents résultats intermédiaires. Par exemple, on peut isoler l'expression mathématique de certains échanges thermiques et en faire une observation locale. Cette orientation, qui correspond à une démarche de recherche fondamentale sera abordée ici, au niveau de la discussion des résultats.

Nous nous sommes, dans cette étude, plutôt attachés à la comparaison des modèles quant aux résultats finaux qu'ils procurent lorsque l'on s'intéresse à l'analyse de la sensation thermique résultant de l'interactivité entre le corps et le milieu physique.

Cette précaution mérite d'être indiquée, car l'observation des échanges thermiques peut aussi s'envisager dans de strictes situations de confort, pour lesquelles l'équilibre des échanges (stabilisés) est réalisé. Ce type d'analyse conduit alors à la comparaison d'indicateurs qui sont généralement des courbes de confort.

Précisons cette idée. Si nous notons A le vecteur ambiance, dont les composantes caractérisent le milieu thermique, nous savons que ce vecteur est défini dans $\mathbb{R}^{4}$, de même que le vecteur $\mathbf{U}$ caractérisant l'usager. De telle manière que l'on peut écrire que la sensation thermique $Y$ résulte de l'opération vectorielle $*$ :

$$
\begin{gathered}
Y=\mathbf{A} * \mathbf{U} \quad \text { avec } \mathbf{A}\left(a_{1} ; a_{2} ; a_{3} ; a_{4}\right) \\
\mathbf{U}\left(u_{1} ; u_{2} ; u_{3} ; u_{4}\right)
\end{gathered}
$$

Les composantes de ces vecteurs sont :

$a_{1}=T_{\mathrm{r}}:$ température radiante moyenne de l'enceinte ;

$a_{2}=T_{\mathrm{a}} \quad$ : température moyenne de l'air à l'intérieur de l'enceinte ;

$a_{3}=V_{\mathrm{a}}$ : vitesse relative de l'air à proximité du sujet ;

$a_{4}=H_{\mathrm{a}}:$ humidité relative moyenne de l'air ;

$u_{1}=m_{\text {th }}$ : métabolisme thermique du sujet;

$u_{2}=R_{\mathrm{v}}$ : résistance thermique de la vêture ;

$u_{3}=F_{\mathrm{v}}$ : facteur de vêture ;

$u_{4}=F_{\mathrm{p}}:$ facteur postural.
Cette sensation thermique $Y$ est un scalaire associé statistiquement à l'expression de la perception thermique de l'ambiance. Son étalonnage fut établi par Fanger au début des années 70, pour les milieux thermiques dits tempérés, et est maintenant reconnue et utilisée à peu près partout dans le monde, sur les sites de recherche où les critères de confort sont pris en compte. En France, l'AFNOR l'a intégrée dans sa norme sur la spécification des conditions de confort thermique. On a la correspondance suivante (Tab. I) :

\begin{tabular}{|c|l|}
\hline Valeur de $Y$ & \multicolumn{1}{|c|}{ Sensation } \\
\hline 3 & Très chaud \\
2 & Chaud \\
1 & Légèrement chaud \\
0 & Neutre \\
-1 & Légèrement froid \\
-2 & Froid \\
-3 & Très froid \\
\hline
\end{tabular}

On notera ici que la plupart des auteurs ramènent le vecteur $\mathbf{U}$ à un vecteur de $\mathbb{R}^{2}$, en admettant que le facteur postural est quasiment constant et que le facteur de vêture est lié à la grandeur $R_{\mathrm{v}}$.

Les deux démarches que nous venons d'évoquer peuvent alors être présentées d'une manière fort simple :

- Le travail que nous présentons ici consiste à comparer les modèles mathématiques sur le critère $Y$ pour lequel la valeur peut être quelconque, en restant dans le domaine de définition admis par la communauté internationale :

$$
Y \in \mathbb{R}, \quad \text { et } \quad Y \in[-3 ;+3]
$$

- La deuxième approche que nous évoquions plus haut consiste, quant à elle, à comparer les modèles dans la situation particulière de la sensation neutre :

$$
Y=0
$$

Nous avons choisi de travailler dans le premier cas afin de mettre en évidence l'intérêt d'un critère $q$ que nous nommerons in ice e qua ite, genere par e critère $Y$. Nous montrerons plus loin que certains domaines d'étude nécessitent ce critère complémentaire, dans le cadre, en particulier, de l'analyse différentielle des projets de bâtiments, en phase de conception.

2.2 MÉTHOde D’ÉTUdE. - Nous avons placé ce travail dans un contexte de connaissance thermique des espaces habitables, en considérant que les résultats obtenus pouvaient alimenter le discours sur les concepts à développer pour l'élaboration d'outils 
automatiques d'aide à la conception. Rappelons ici brièvement que ces outils comportent essentiellement, dans les modules d'analyse thermique, des programmes de calcul conduisant à l'évaluation de deux critères, celui de la consommation énergétique et celui de la qualité des ambiances. Nous avons donc voulu montrer quelles pouvaient être les conséquences du choix de tel ou tel modèle sur les décisions induites, dans le domaine de la qualité thermique.

Nous avons convenu d'un protocole de comparaison, consistant à se placer en situation d'opérationnalité, c'est-à-dire en situation d'exploitation des modèles en avant-projet. Cela nous a conduit·à simuler la sensation thermique de l'usager $(Y)$ avec chacun d'eux, sur différentes séquences climatiques, pour un exemple d'objet bâtiment.

Ce n'est, en effet, que sur une séquence climatique que l'on peut correctement observer les écarts entre les modèles. L'étude figée, à un instant donné ne présente qu'un intérêt limité, compte tenu des objectifs visés. La figure 1 résume la méthode d'étude que nous avons employée.

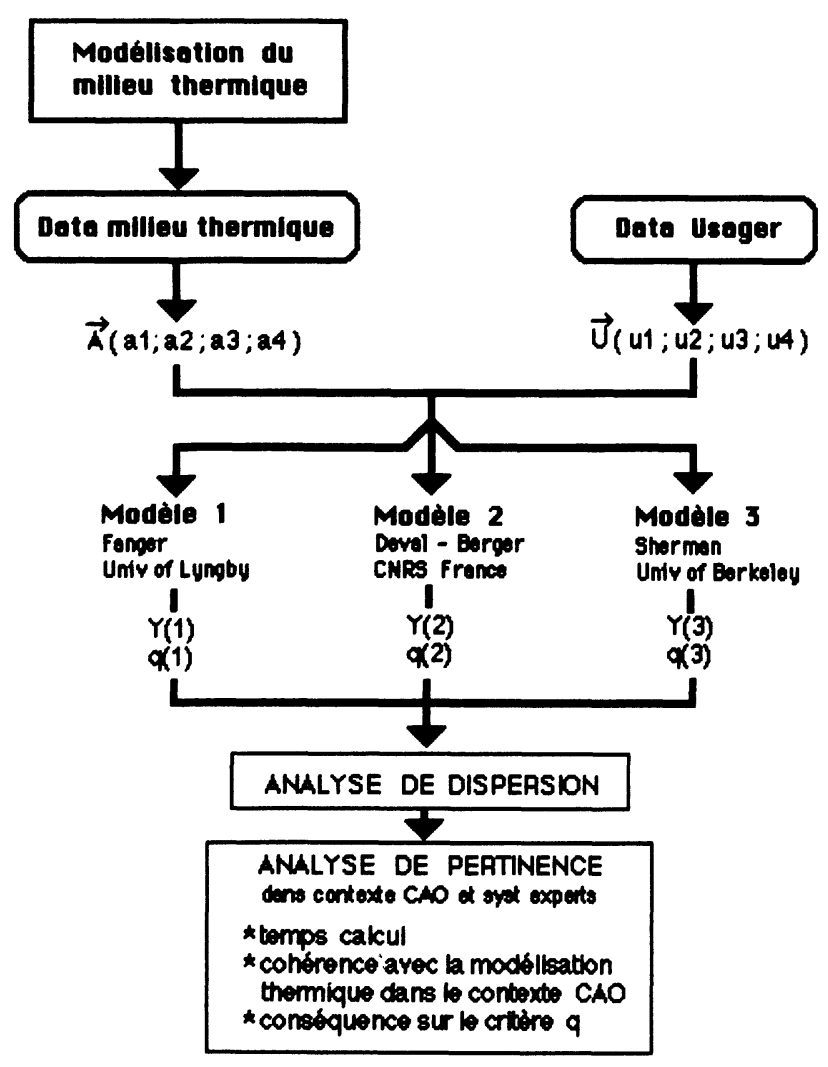

Fig. 1. - Schéma explicitant notre méthode d'étude.

[Sketch describing our method.]

Au plan de la chronologie de ce travail, nous nous sommes appuyés sur les résultats d'une étude d'ambiances en saison chaude, publiée en 1985 [4].
Cette étude nous a fourni la distribution temporelle du vecteur ambiance $\mathbf{A}\left(t, a_{i}\right)$.

Nous avons ensuite admis que le vecteur $\mathbf{U}$ conservait les mêmes composantes dans le temps, c'est-à-dire que :

$$
\mathbf{U}\left(t, u_{i}\right)=\mathbf{U}\left(u_{i}\right) .
$$

Dans cette étude, nous avons considéré un individu dont les caractéristiques étaient :

$$
\begin{gathered}
m=m_{\text {th }}=58,1 \mathrm{~W} / \mathrm{m}^{2} \text { soit } a=1 \text { (assis au repos) } \\
R_{\mathrm{v}}=0,155 \mathrm{~m}^{2}{ }^{\circ} \mathrm{C} / \mathrm{W} .
\end{gathered}
$$

Le traitement des vecteurs $\mathbf{A}$ et $\mathbf{U}$ par les différents modèles nous a ensuite conduit à comparer le critère $Y(X)$ obtenu.

Finalement, comme nous venons de l'indiquer, $Y(X)$ générant un deuxième critère $\dot{q}(X)$ que nous présenterons plus loin, nous nous sommes intéressés à la dispersion des résultats et surtout aux conséquences de l'emploi de tel ou tel modèle (résultats optimistes, pessimistes...). Mais un deuxième propos est ensuite développé sur le choix des modèles et la cohérence de ce choix avec celui des outils permettant d'accéder au vecteur $\mathbf{A}$, c'est-à-dire les programmes de calcul exécutant la simulation du comportement thermique des enceintes, en amont de notre calcul.

\section{Présentation des modèles d'échanges thermiques.}

3.1 Remarque préliminaire. - Afin de comparer plus aisément les résultats obtenus, nous avons choisi le parti de présenter les modèles simultanément, sur un mode d'échange thermique donné. A cet effet, rappelons brièvement que le corps utilise 7 modes d'échanges thermiques qui sont :

- la convection (libre et parfois forcée) (CV); IR)

- le rayonnement (grandes longueurs d'ondes

- la convection respiratoire

(ry) ;

la perspiration (t

- le transfert d'humidité respiratoire (hr);

- la sudation

(sd) ;

- la conduction

Indiquons toutefois que le mode conductif est généralement ignoré, car :

- lorsque le sujet est debout, ce transfert se limite à celui de ses semelles. Dans la plupart des cas courants, il est négligé. Il exige toutefois des travaux très spécialisés dans les cas des sols froids ou des sols chauds (planchers chauffants),

- lorsque le sujet est assis, la prise en compte de la conduction donnerait alors lieu à des études de transferts couplés où l'inertie thermique du siège devrait être prise en compte. Comme le sujet va probablement adopter une attitude variable (se levant et se rasseyant), une simulation fine de ces 
mécanismes ne serait pas cohérente avec les objectifs visés, mais aussi avec la précision des méthodes de calcul qui permettent d'atteindre le vecteur $\mathbf{A}\left(t, a_{i}\right)$.

En principe, on propose, pour tenir compte de l'effet du siège, de majorer artificiellement la résistance de vêture de 20 à $40 \%$.

D'une manière générale, nous ne donnerons pas, dans les paragraphes suivants, de justification physique des modèles que nous présentons et comparons. On pourra se reporter, pour cela, aux publications d'origine des auteurs qui sont assez complètes sur le sujet [1], [2], [3].

3.2 EChANGeS CONVECTIFS $E_{\mathrm{CV}}(X)$. - Dans la plupart des situations, le corps baigne dans un fluide (l'air de l'ambiance) avec lequel il a des échanges convectifs. Ces échanges peuvent, en première approximation et compte tenu des objectifs des modèles, se mettre sous la forme générale d'une densité de flux $E_{\mathrm{CV}}$ traduisant une répartition du transfert de chaleur total en 3 zones :

- la tête (indice t) ;

- les mains (indice $\mathrm{m}$ ) ;

- le reste du corps, c'est-à-dire la partie vêtue (indice $\mathrm{v}$ )

$$
E_{\mathrm{CV}}=e_{\mathrm{CV}} \text { (p. vêtue) }+e_{\mathrm{CV}} \text { (tête) }+e_{\mathrm{CV}} \text { (mains) } \text {. }
$$

Chacune des densités de flux composites obéissent alors à une loi du type :

$$
e_{\mathrm{CV}}=\alpha F h_{\mathrm{C}}\left(T_{\mathrm{s}}-T_{\mathrm{a}}\right)
$$

où :

$\alpha$ est le coefficient de proportionnalité de la densité du flux transitant par chacune des zones (tête, mains, partie vêtue);

$F$ est un coefficient permettant éventuellement de majorer l'échange thermique : c'est principalement le cas de la partie vêtue, pour laquelle on admet que le vêtement majore la surface d'échange [1], [2] ;

$h_{\mathrm{C}}$ est le coefficient d'échange convectif, fonction

— du régime d'écoulement de l'air autour du corps,

- de la vitesse relative de l'air,

- de la température de la surface (vêtement, peau si a convection peut etre consı erée comme naturelle

$T_{\mathrm{s}}$ est la température de la surface du corps concernée par l'échange : vêtement ou peau ;

$T_{\mathrm{a}}$ est la température sèche de l'air de l'ambiance.

Avec les conventions de notations adoptées, nous avons, pour le modèle $X$ :

$$
\begin{aligned}
E_{\mathrm{CV}}(X)=\alpha_{\mathrm{v}, X} F_{\mathrm{v}, X} & h_{\mathrm{C}, \mathrm{v}, X}\left(T_{\mathrm{v}, X}-T_{\mathrm{a}}\right)+ \\
& +\alpha_{\mathrm{t}, X} h_{\mathrm{C}, \mathrm{t}, X}\left(T_{\mathrm{c}, X}-T_{\mathrm{a}}\right)+\alpha_{\mathrm{m} X} \\
& \times h_{\mathrm{C}, \mathrm{m}, X}\left(T_{\mathrm{c}, X}-6-T_{\mathrm{a}}\right) .
\end{aligned}
$$

3.2.1 Le facteur de vêture $F_{\mathrm{v}, X}$ - On a :

$F_{\mathrm{v}, 1}=1+0,77 \cdot R_{\mathrm{v}}$

$F_{\mathrm{v}, 2}=F_{\mathrm{v}, 1}$

$F_{\mathrm{v}, 3}=\left(1+0,23 I_{\mathrm{cle}}\right) /\left(1+0,178 I_{\mathrm{cle}}\left[h_{\mathrm{C}, 3}+h_{\mathrm{r}, 3}\right]\right)$

avec :

$I_{\mathrm{cle}}=6,45\left[R_{\mathrm{v}}+\left(1-F_{\mathrm{v}, 1}\right)\right] /\left[\left(h_{\mathrm{C}, 3}+h_{\mathrm{r}, 3}\right) F_{\mathrm{v}, 1}\right] \approx$ $\approx 5,56 R_{\mathrm{v}}$.

3.2.2 Les coefficients de partition des échanges $\alpha_{\mathrm{v}, X}, \alpha_{\mathrm{t}, X}, \alpha_{\mathrm{m}, X} \cdot-\mathrm{Ce}$ sont les coefficients qui pondèrent les échanges convectifs des différentes parties du corps. Fanger et Sherman négligent cette pondération, qui n'a été que récemment introduite par Deval et Berger [2]. On a donc :

$$
\begin{aligned}
& \alpha_{\mathrm{v}, 1}=\alpha_{\mathrm{v}, 3}=1 \quad \alpha_{\mathrm{v}, 2}=0,94 \quad \text { (partie vêtue) } \\
& \alpha_{\mathrm{t}, 1}=\alpha_{\mathrm{t}, 3}=0 \quad \alpha_{\mathrm{t}, 2}=0,02 \quad \text { (tête) } \\
& \alpha_{\mathrm{m}, 1}=\alpha_{\mathrm{m}, 3}=0 \quad \alpha_{\mathrm{m}, 2}=0,04 \quad \text { (mains). }
\end{aligned}
$$

3.2.3 Les coefficients d'échanges convectifs. 1) Partie vêtue :

$$
\begin{aligned}
h_{\mathrm{C}, \mathrm{v}, 1} & =\sup \left[2,38\left|T_{\mathrm{v}, 1}-T_{\mathrm{a}}\right|^{0,25} ; 12,06 \sqrt{V_{\mathrm{a}}}\right] \\
h_{\mathrm{C}, \mathrm{v}, 2} & =\sup \left[2,38\left|T_{\mathrm{v}, 2}-T_{\mathrm{a}}\right|^{0,25} ; 10 \sqrt{V_{\mathrm{a}}}\right] \\
h_{\mathrm{C}, 3} & =\sup \left[5,66\left(\frac{m}{m_{0}}-0,85\right)^{0,39} ; 8,3 V_{\mathrm{a}}^{0,53}\right] .
\end{aligned}
$$

2) Partie tête :

$$
\begin{aligned}
& h_{\mathrm{C}, \mathrm{t}, 1} \text { et } h_{\mathrm{C}, \mathrm{t}, 3} \text { non définis }\left(\alpha_{\mathrm{t}, 1}=\alpha_{\mathrm{t}, 3}=0\right) \\
& h_{\mathrm{C}, \mathrm{t}, 2}=\sup \left[2,38\left|T_{\mathrm{c}, 2}-T_{\mathrm{a}}\right|^{0,25} ; 10 \sqrt{V_{\mathrm{a}}}\right] .
\end{aligned}
$$

3) Partie mains :

$$
\begin{aligned}
& h_{\mathrm{C}, \mathrm{m}, 1} \text { et } h_{\mathrm{C}, \mathrm{m}, 3} \text { non définis }\left(\alpha_{\mathrm{m}, 1}=\alpha_{\mathrm{m}, 3}=0\right) \\
& h_{\mathrm{C}, \mathrm{m}, 2}=\sup \left[2,38\left|T_{\mathrm{c}, 2}-6-T_{\mathrm{a}}\right|^{0,25} ; 10 \sqrt{V_{\mathrm{a}}}\right]
\end{aligned}
$$

3.2.4 Température $T_{\mathrm{v}, X}$ et $T_{\mathrm{c}, X}$ - - Il s'agit de la température de surface extérieure de la vêture et de la température cutanée.

1) $T_{v, 1}$ et $T_{v, 2}$ (modèles de Fanger et Deval) ne peuvent être calculées directement, compte tenu des transferts couplés avec l'état d'ambiance. Toutefois, une simple itération, en principe ultra rapide, permet d'obtenir ces grandeurs.

Quant au modèle de Sherman, nous y reviendrons plus loin, un recalage de ses expressions lui permet d'écrire :

$$
T_{\mathrm{v}, 3}=T_{\mathrm{c}, 3} .
$$

2) Pour les températures cutanées, on a :

$$
\begin{aligned}
& T_{\mathrm{c}, 1}=3 \dot{5}, 7-0,0275 m_{\mathrm{th}} \\
& T_{\mathrm{c}, 2}=29,55+0,196 T_{\mathrm{A}}-1,064 \frac{m}{m_{0}}\left(1-1,9 R_{\mathrm{v}}\right) \\
& T_{\mathrm{c}, 3}=T_{\mathrm{c}, 1}
\end{aligned}
$$


$T_{\mathrm{A}}$ est une température résultante définie par:

$$
T_{\mathrm{A}}=\left(h_{\mathrm{C}, \mathrm{v}, 2} \cdot T_{\mathrm{a}}+h_{\mathrm{r}, \mathrm{v}, 2} \cdot T_{\mathrm{r}}\right) /\left(h_{\mathrm{C}, \mathrm{v}, 2}+h_{\mathrm{r}, \mathrm{v}, 2}\right) .
$$

On notera que $T_{\mathrm{c}, 1}$ et $T_{\mathrm{c}, 3}$ pourront être calculées directement à partir des données d'entrée alors que $T_{\mathrm{c}, 2}$ exigera une itération.

3.3 ECHANGES RADIATIFS $E_{\mathrm{r} y}(X)$. - Les échanges radiatifs entre la surface du corps et l'ambiance sont certainement ceux que l'on perçoit le moins intuitivement, du fait qu'ils se développent dans le domaine de l'infrarouge. L'équation de base de ces transferts traduit l'expression du flux net échangé entre la surface du corps et l'ensemble des autres surfaces constituant l'environnement: parois du local si le sujet est dans un habitacle.

Cet échange, rappelons-le, néglige l'absorption radiative de l'air et dépend:

- de l'aire et de la position relative des surfaces échangeant du rayonnement ;

— de l'émissivité et du coefficient d'absorption de chacune des surfaces;

- de leurs températures.

Ils s'écrivent, suivant les modèles :

$$
\begin{gathered}
E_{\mathrm{r} y}(1)=F_{\mathrm{v}, 1} \cdot F_{\mathrm{p}, 1} h_{\mathrm{r}, 1} \times \\
\times\left[\left(T_{\mathrm{v}, 1}+273\right)^{4}-\left(T_{\mathrm{r}}+273\right)^{4}\right] \\
E_{\mathrm{r} y}(2)=F_{\mathrm{p}, 2}\left[0,94 F_{\mathrm{v}, 2} h_{\mathrm{r}, \mathrm{v}, 2}\left(T_{\mathrm{v}, 2}-T_{\mathrm{r}}\right)+\right. \\
+0,02 h_{\mathrm{r}, \mathrm{t}, 2}\left(T_{\mathrm{c}, 2}-T_{\mathrm{r}}\right) \\
\left.+0,04 h_{\mathrm{r}, \mathrm{m}, 2}\left(T_{\mathrm{c}, 2}-6-T_{\mathrm{r}}\right)\right] \\
E_{\mathrm{ry}}(3)=F_{\mathrm{v}, 3} h_{\mathrm{r}, 3}\left(T_{\mathrm{c}, 3}-T_{\mathrm{r}}\right) .
\end{gathered}
$$

3.3.1 Facteur postural $F_{\mathrm{p}, X}$. - C'est un facteur rendant compte du facteur de forme corps-ambiance $F_{\mathrm{p}, 1}=F_{\mathrm{p}, 2}=0,71$.

Il s'agit là d'une valeur moyenne commune à la position debout et à la position assise. Cette approximation est généralement acceptée par les différents chercheurs.

$$
\begin{aligned}
& \text { 3.3.2 Coefficients d'échange radiatif. } \\
& h_{\mathrm{r}, 1}=5,56 \mathrm{~W} / \mathrm{m}^{2}{ }^{\circ} \mathrm{C} \\
& h_{\mathrm{r}, \mathrm{v}, 2}=22.10^{-8}\left[\frac{T_{\mathrm{v}, 2}+T_{\mathrm{r}}}{2}+273\right]^{3}
\end{aligned}
$$

(émissivité de la vêture : 0,97)

$$
\begin{aligned}
h_{\mathrm{r}, \mathrm{t}, 2} & =22,7 \cdot 10^{-8}\left[\frac{T_{\mathrm{c}, 2}+T_{\mathrm{r}}}{2}+273\right]^{3} \\
h_{\mathrm{r}, \mathrm{m}, 2} & =22,7 \cdot 10^{-8}\left[\frac{T_{\mathrm{c}, 2}-6+T_{\mathrm{r}}}{2}+273\right]^{3} \\
h_{\mathrm{r}, 3} & =4,7 \mathrm{~W} / \mathrm{m}^{2}{ }^{\circ} \mathrm{C} .
\end{aligned}
$$

3.4 EChANGES PAR PERSPIRATION $E_{\mathrm{ps}}(X)$. - La perspiration est un mécanisme de transfert insensible de vapeur au travers des couches de l'épiderme. La vapeur perspirée entraîne une puissance calorifique correspondant à la chaleur potentielle de l'eau évaporée. Le corps évacue ainsi une quantité non négligeable de chaleur, et qui oblige l'individu à absorber un minimum de liquide sous la forme de boissons diverses.

Ces échanges thermiques, sous forme latente, ont des expressions assez différentes suivant les auteurs. On a :

$$
E_{\mathrm{ps}}(1)=0,41\left[43,2-0,052 m_{\mathrm{th}}-p_{\mathrm{v}}^{*}\left(T_{\mathrm{a}}\right)\right]
$$

où $p_{\mathrm{v}}^{*}\left(T_{\mathrm{a}}\right)$ est la pression de vapeur de l'air de l'ambiance, exprimée en $\mathrm{mmHg}$

$$
E_{\mathrm{ps}}(2)=0,41\left[p_{\mathrm{vs}}\left(T_{\mathrm{c}, 2}\right)-p_{\mathrm{v}}\left(T_{\mathrm{a}}\right)\right]
$$

où $p_{\mathrm{vs}}\left(T_{\mathrm{c}, 2}\right)$ est la pression de vapeur saturante à la surface de la peau

$$
E_{\mathrm{ps}}(3)=0,06\left[E_{\max }-E_{\mathrm{sd}}(3)\right]
$$

où $E_{\max }=2.2 h_{\mathrm{C}, 3} F_{\mathrm{v}, \mathrm{c}, 3}\left[p_{\mathrm{vs}}^{*}\left(T_{\mathrm{c}, 3}\right)-p_{\mathrm{vs}}^{*}\left(T_{\mathrm{d}}\right)\right]$, les pressions de vapeur saturante étant ici exprimées en torr. Sherman montre qu'il peut approximer cet écart de pression de vapeur saturante par : ( $T_{\mathrm{d}}$ est la température de rosée de l'air ambiant)

$$
\begin{gathered}
p_{\mathrm{vs}}^{*}\left(T_{\mathrm{c}, 3}\right)-p_{\mathrm{vs}}^{*}\left(T_{\mathrm{d}}\right)=\left(T_{\mathrm{c}, 3}^{2}-T_{\mathrm{d}}^{2}\right) / T_{\mathrm{c}, 3} \\
F_{\mathrm{v}, \mathrm{e}, 3}=\left(1+0,143 h_{\mathrm{C}, 3} \cdot I_{\mathrm{cle}}\right)^{-1}
\end{gathered}
$$

et $E_{\mathrm{sd}}(3)=0,42 m_{0}(a-1)$ qui est la quantité de chaleur sudée. $a$ représente l'activité : $a=m / m_{0}$.

3.5 ECHANGeS RESPIRATOIRES HUMIDES $E_{\mathrm{hr}}(X)$. - La vapeur d'eau rejetée par notre respiration représente un petit potentiel de chaleur. Comme dans le cas de la perspiration, cette vapeur véhicule sa chaleur de vaporisation et contribue à refroidir légèrement le corps

$$
\begin{aligned}
& E_{\mathrm{hr}}(1)=0,0023 m\left[44-p_{\mathrm{v}}\left(T_{\mathrm{a}}\right)\right] \\
& E_{\mathrm{hr}}(2)=0,0023 m\left[p_{\mathrm{vs}}\left(T_{\text {exp }}\right)-p_{\mathrm{v}}\left(T_{\mathrm{a}}\right)\right]
\end{aligned}
$$

où $T_{\text {exp }}$ est la température sèche de l'air expiré

$$
T_{\text {exp }}=32.6+0,066 T_{\mathrm{a}}+0,032 w_{\mathrm{a}}
$$

$w_{\mathrm{a}}$ : humidité absolue de l'air ambiant

$$
E_{\mathrm{hr}}(3)=0,0023 m\left(T_{\mathrm{c}, 3}^{2}-T_{\mathrm{d}}^{2}\right) / T_{\mathrm{c}, 3} .
$$

3.6 ECHANGES RESPIRATOIRES CONVECTIFS $E_{\mathrm{c}, \mathrm{r}}(X)$. - Il s'agit là du flux de chaleur sensible que véhicule l'air expiré et qui est libéré par les poumons. C'est un mode d'échange relativement mineur. 
Facile à quantifier, il est pris en compte de la manière suivante par les différents auteurs :

$$
\begin{aligned}
& E_{\mathrm{cr}}(1)=0,0014 m\left(34-T_{\mathrm{a}}\right) \\
& E_{\mathrm{cr}}(2)=0,0014 m\left(T_{\exp }-T_{\mathrm{a}}\right) \\
& E_{\mathrm{cr}}(3)=0,0014 m\left(T_{\mathrm{c}, 3}-T_{\mathrm{a}}\right) .
\end{aligned}
$$

3.7 ECHANGES PAR SUDATION $E_{\text {sd }}(X)$. - La sudation est un mécanisme thermorégulateur particulier. La sueur secrétée à la surface du corps consomme une part de la chaleur métabolique pour s'évaporer : il s'agit donc, comme pour la perspiration, d'un transfert de chaleur et de masse, du corps vers l'ambiance.

L'analyse fine du phénomène de sudation (rythme, répartition, ...) est délicate et la communauté mondiale des thermophysiologistes n'est pas tombée d'accord sur toutes les questions posées : capacité de résorption de l'ambiance, distribution inhomogène du film liquide, rendement d'évaporation du corps, notions de mouillure, de ruissellement, ...

$\mathrm{Au}$ regard de ce contexte, les 3 auteurs étudiés utilisent la même loi de sudation :

$$
E_{\mathrm{sd}}(1)=0,42\left(m_{\mathrm{th}}-58,1\right)=E_{\mathrm{sd}}(2)=E_{\mathrm{sd}}(3) .
$$

Notons que cette expression des échanges par sudation a été établie pour un individu en situation de neutralité thermique. La question de la sudation est assez complexe et les études poussées sur le sujet montrent qu'elle est délicate à relier à la notion de confort.

Givoni (cité dans [5]) propose que la situation du sujet puisse être éventuellement considérée comme confortable (compte tenu des autres échanges) tant que : $E_{\text {sd }}(1) \leqslant 47 / S_{\text {c }}$.

Dans le travail que nous présentons ici, nous avons vu que les sujets pourraient se trouver en situation de déséquilibre thermique, c'est-à-dire que $Y$ peut varier entre -3 et +3 . La quantité $E_{\text {sd }}(X)$ est alors un reliquat, dans le bilan des échanges, dont la formulation exacte ne nous est, de fait, pas nécessaire. Quoi qu'il en soit, dans le cadre de cette étude comparative, les auteurs ont adopté la même expression.

\section{Sensation thermique et indice de qualité.}

4.1 Sensation thermiQue. - Le bilan des échanges hors sudation, pour les modèles 1 et 2 s'écrira simplement :

$$
\begin{aligned}
E(X)=E_{\mathrm{CV}}(X)+E_{\mathrm{ry}}(X)+E_{\mathrm{ps}}(X)+ \\
+E_{\mathrm{hr}}(X)+E_{\mathrm{cr}}(X) .
\end{aligned}
$$

La sudation (ou le frisson) se déclenchera si :

$$
m_{\mathrm{th}} \neq E(X) \text {. }
$$

On dégage alors la notion de différentiel thermique, à un instant $t$ de la séquence d'observation :

$$
D(X, t)=m_{\mathrm{th}}(t)-E^{*}(X, t)
$$

où $E^{*}(X, t)$ est la somme algébrique des échanges calculés à l'instant $t$ incluant la sudation de confort calculée au paragraphe 3.7

$$
E^{*}(X, t)=E(X, t)+E_{\mathrm{sd}}(X, t) .
$$

La sensation thermique probable que percevra l'usager sera :

$$
Y(X, t)=L(m) . D(X, t)
$$

où $L(m)$ est une fonction d'ajustement d'origine expérimentale :

$$
L(m)=0,303 \mathrm{e}^{-0,0362 m}+0,028 .
$$

Le modèle de Sherman, quant à lui, présente l'originalité de proposer un calcul direct (sans itération) de la sensation. On lui doit en effet le formalisme :

$Y(3, t)=Y_{0}+Y_{\mathrm{ry}} \cdot \frac{T_{\mathrm{r}}(t)}{T_{\mathrm{c}, 3}}+Y_{\mathrm{CV}} \cdot \frac{T_{\mathrm{a}}(t)}{T_{\mathrm{c}, 3}}+Y_{\mathrm{e}} \cdot \frac{T_{\mathrm{d}}^{2}(t)}{T_{\mathrm{c}, 3}^{2}}$

où $Y_{0}, Y_{\mathrm{ry}}, Y_{\mathrm{CV}}$ et $Y_{\mathrm{e}}$ sont des coefficients dont les expressions sont données en annexe.

Notons au passage que, si le métabolisme $m$ devient une fonction du temps, ce qui se produit en général dans la réalité, alors les valeurs de $Y$ et $T_{\mathrm{c}, 3}$ deviennent aussi dépendantes de $t$.

4.2 INDICE DE QUALITÉ. - Afin de pouvoir comparer des objets entre eux, nous avons relié la sensation thermique à un indice de satisfaction établi statistiquement par le groupe du Pr Fanger. Il s'agit du pourcentage prévisible de satisfaits (Predicted percentage of satisfied) que nous noterons $q$ :

$$
q(Y)=\mathrm{e}^{K(Y)}
$$

avec :

$$
K(Y)=4,558+0,0018 Y-0,2552 Y^{2} .
$$

Lorsque nous travaillons sur une séquence $n \cdot \Delta t$ donnée, on est conduit à traiter les indices de satisfaction calculés à chaque pas de temps $t$. Finalement, pour un mo e e onne, ce a consiste à étudier la fonction $q(X, t)$ :

$$
q(X, t)=\mathrm{e}^{K[Y(X, t)]} .
$$

\section{Présentation des résultats obtenus.}

5.1 Définition DU Vecteur $\mathbf{A}\left(u_{i}, t\right)$. - Nous avons indiqué plus haut que notre comparaison des modèles avait été conçue en intégrant la variable temporelle. Nous avons considéré un système physique bâtiment donné, dont le traitement en simula- 
tion numérique conduisait à la définition du vecteur ambiance, $\mathbf{A}\left(u_{i}, t\right)$, à chaque instant $t=n . \Delta t$.

Nous donnons ci-après sa définition aux figures 2 et 3 .

Nous avons traité 2 séquences :

- la première est une séquence du mois de juillet, relativement douce ;

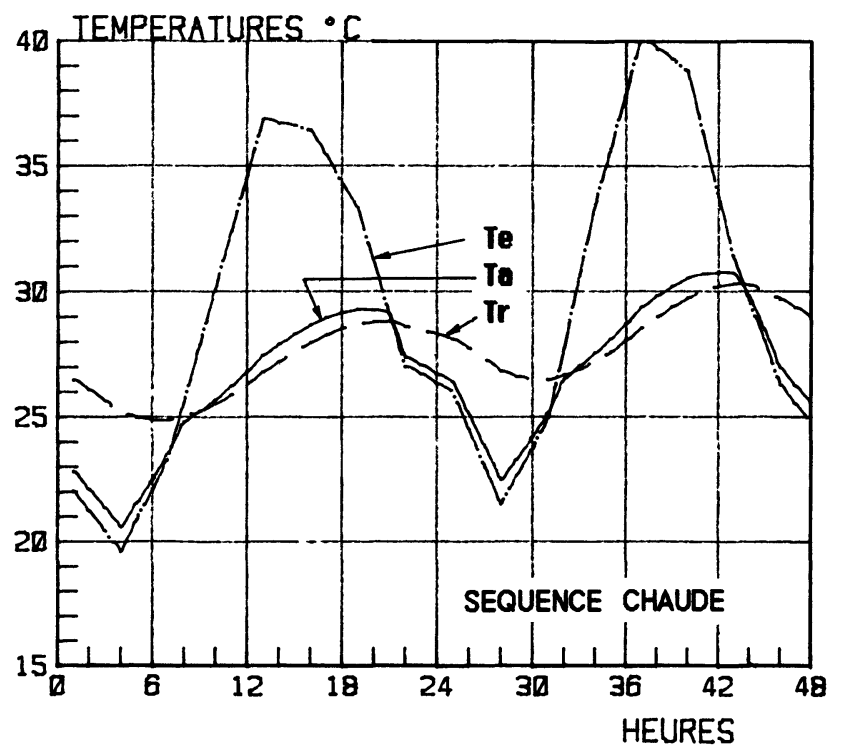

- la deuxième est aussi une séquence du mois de juillet, mais beaucoup plus chaude (notée séquence chaude sur les diagrammes);

On notera que la vitesse de l'air présente des variations de type échelon. Cela provient du fait que cette composante de A n'est pas calculée par le programme de simulation thermique. On admet

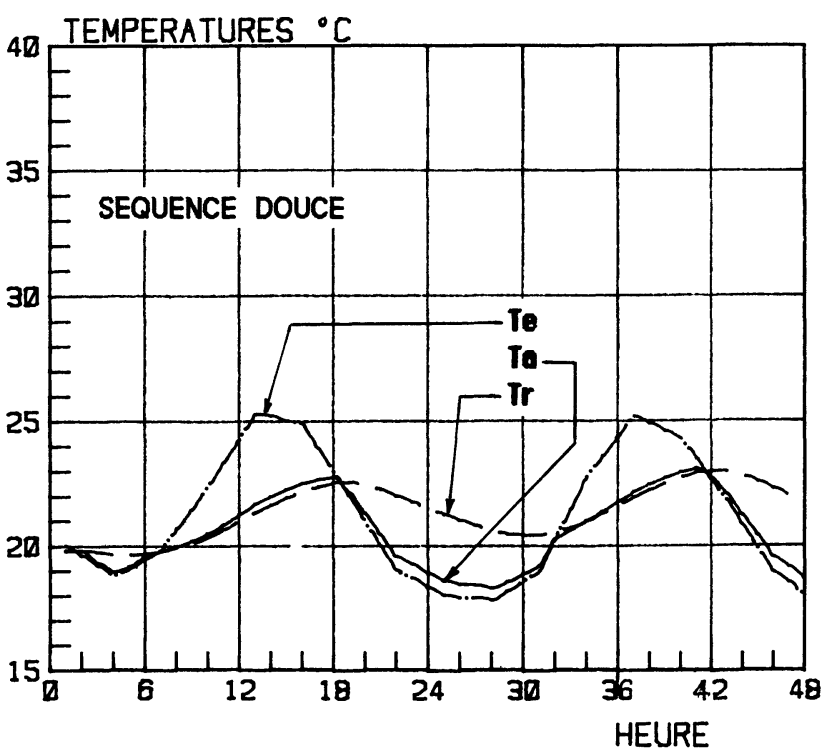

Fig. 2. - Composantes du vecteur $\mathbf{A}\left(u_{i}, t\right)$. La température extérieure a été rajoutée sur ces diagrammes (3 et 4 juillet puis 9 et 10 juillet).

[Components of the vector $\mathbf{A}\left(u_{i}, t\right)$. The outdoor temperature has been added on the diagrams (July 3th, 4th for warm sequence ; July 9 th, 10 th for hot sequence).]
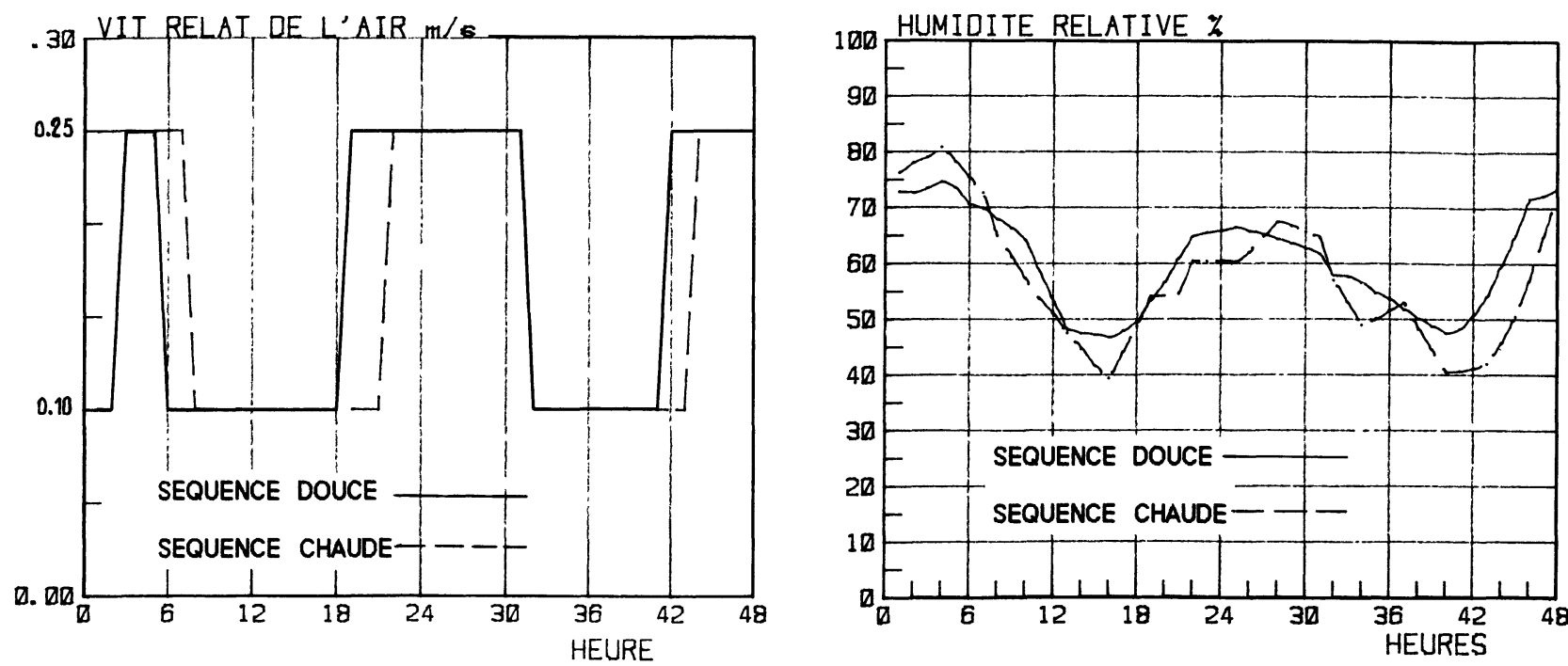

Fig. 3. - Composantes du vecteur $\mathbf{A}\left(u_{i}, t\right)$. La valeur de $V_{\mathrm{a}}(t)$ n'est pas calculée dans la simulation numérique, mais déterminée par un test comparatif de température (Ext-Int) et traduit l'attitude de l'usager qui ouvre éventuellement les fenêtres.

[Components of the vector $\mathbf{A}\left(u_{i}, t\right)$. Value of air velocity $V_{\mathrm{a}}(t)$ is not obtained with numerical simulation, but determined by a temperature comparative test (outdoor-indoor) and indicate if the habitant open windows.] 
simplement que, lorsque les fenêtres sont ouvertes, l'usager est soumis à un front de vitesse de $0,25 \mathrm{~m} / \mathrm{s}$, valeur moyenne calculée à partir des débits traversants. Il s'agit là d'une approximation. Toutefois, celle-ci paraît raisonnable puisque lorsque cette vitesse atteint $0,3 \mathrm{~m} / \mathrm{s}$, il $\mathrm{y}$ a déclenchement du phénomène de gêne mécanique et régulation manuelle par l'usager, qui va limiter l'ouverture des fenêtres. Lorsqu'elles sont complètement fermées, on admet que $V_{\mathrm{a}} \approx 0,1 \mathrm{~m} / \mathrm{s}$ (vitesses induites par une VMC).

Le choix de ces deux séquences, à la même saison, va nous permettre de comparer des modèles dans des situations assez différentes. Par ailleurs, nous nous sommes limités à la saison chaude dans la mesure où c'est pendant cette période que l'inconfort peut apparaître. L'hiver et la demi-saison réduisent les écarts entre modèles, en termes de sensation thermique, car l'on se trouve la plupart du temps dans la situation de neutralité $(Y=0)$, du fait de la régulation.

5.2 REMARQUES PRÉLIMINAIRES SUR LES MODÈLES. - Les 3 modèles que nous avons exploités sont, en fait assez proches :

- Le modèle du groupe du Pr Franger est actuellement le modèle le plus répandu dans le monde pour l'étude des sensations thermiques. Un modèle plus sophistiqué existe et est notamment utilisé par la NASA pour la prédiction des ambiances dans l'espace habitable embarqué (Stolwick [6]). Toutefois, compte tenu de la précision avec laquelle nous simulons le comportement thermique des cellules habitées au sol et déterminons le vecteur d'entrée A, il est actuellement acceptable de rechercher la sensation thermique à l'aide du modèle de Fanger.

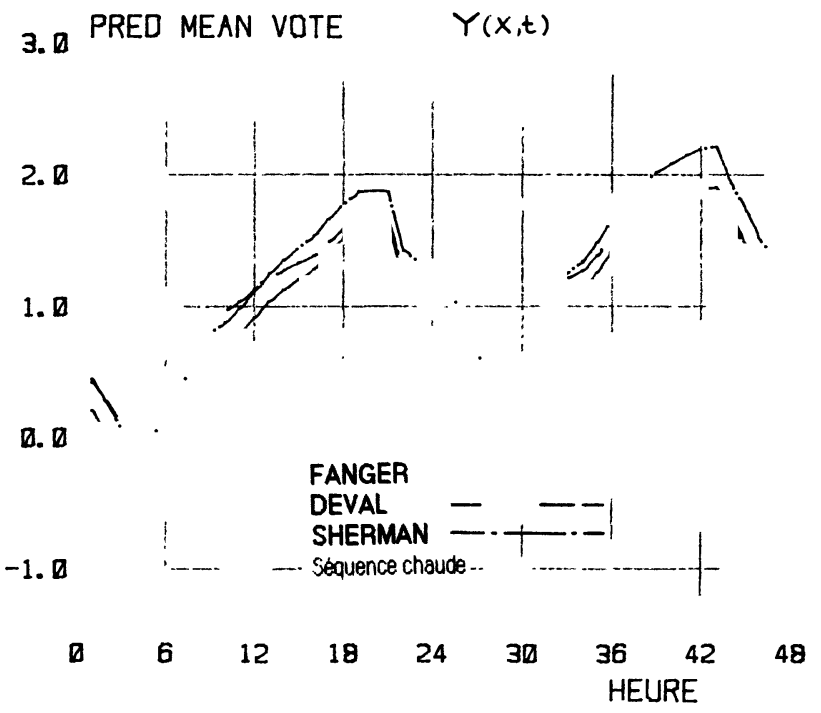

Nous le considérerons ici comme le modèle de référence.

- Le modèle de Deval et Berger est très proche et très inspiré de celui de Fanger. En fait, il en est une version modifiée, comportant des variations qui doivent en améliorer la précision. Ainsi, le corps est décomposé en 3 parties (tête, mains et partie vêtue) dont chacune possède sa propre température de surface, alors que chez Fanger, le corps est considéré globalement, avec une température de surface unique.

- Le modèle de Sherman est, aussi, déduit de celui de Fanger. Il ne s'agit donc pas, à proprement parler, d'un modèle nouveau, car les mécanismes thermophysiologiques sont traités de la même manière. La nouveauté provient de l'introduction d'un nouvel algorithme supprimant les calculs itératifs. Nous renvoyons le lecteur à la bibliographie pour plus ample information.

\subsection{COMPARAISON DES RÉSULTATS SUR LA SENSA-} TION THERMIQUE. - Les diagrammes de la figure 4 montrent comment évoluent les fonctions $Y(X, t)$.

* Sur la séquence douce, on constate que les modèles de Fanger et de Sherman conduisent à peu près au même résultat: les sensations thermiques prédites sont presque toujours identiques. Par contre, le modèle de Deval aboutit à des résultats assez différents. Ainsi, à $19 \mathrm{~h}$, par exemple, les sensations obtenues sont inverses. On note aussi que, pendant cette séquence, l'écart est à peu près permanent :

$$
Y(2, t)-Y(1, t) \approx 0,5, \quad \forall t .
$$

Ainsi, on aboutit à une dérive du modèle vers les sensations plus chaudes par rapport à la référence.

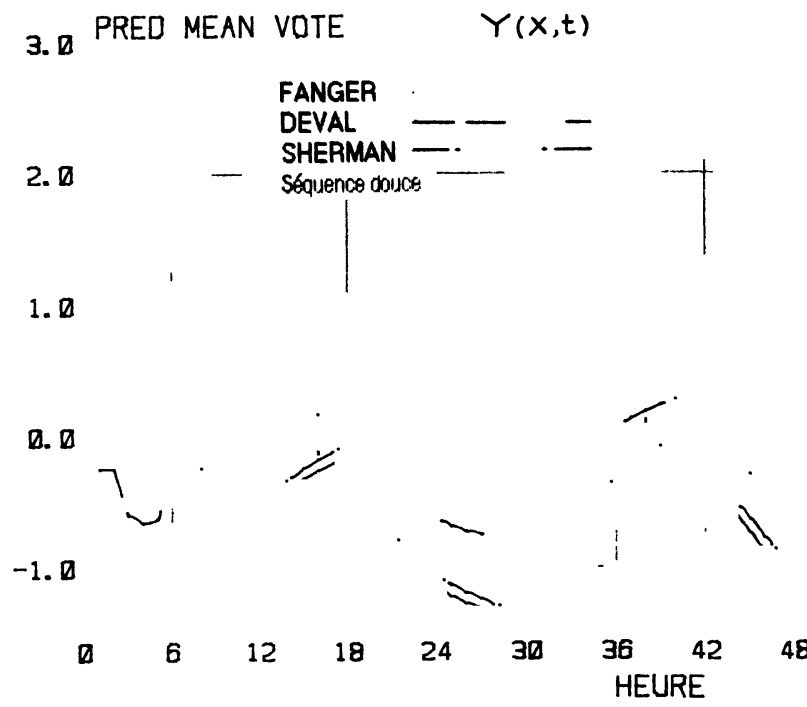

Fig. 4. - Résultats obtenus sur la sensation thermique : fonction $Y(X, t)$.

[Results obtained on thermal feeling : function $Y(X, t)$.] 
* Sur la séquence chaude, les résultats se resserrent et l'on reste à l'intérieur d'un faisceau d'amplitude 0,25 sur l'échelle de la sensation $Y$

$$
\Delta Y(t) \approx 0,25 \text {. }
$$

Les tendances s'inversent puisque c'est le modèle de Sherman qui diverge le plus par rapport à la référence. Le modèle de Deval paraît ici beaucoup plus proche de celui de Fanger. Il semblerait donc qu'il soit plus adapté aux séquences chaudes, si nous admettons le principe d'un recalage par rapport à Fanger. La valeur objective de chacun de ces modèles ne peut, bien entendu, être discutée que par rapport à l'expérimentation.

A ce sujet, la référence accordée au modèle de Fanger peut être discutée, car il a été mis au point sur des ambiances qui sont plutôt celles des climats tempérés. La séquence chaude n'est plus vraiment dans cette catégorie. Nous admettons toutefois que la référence, en termes stricts de comparaison, est maintenue.
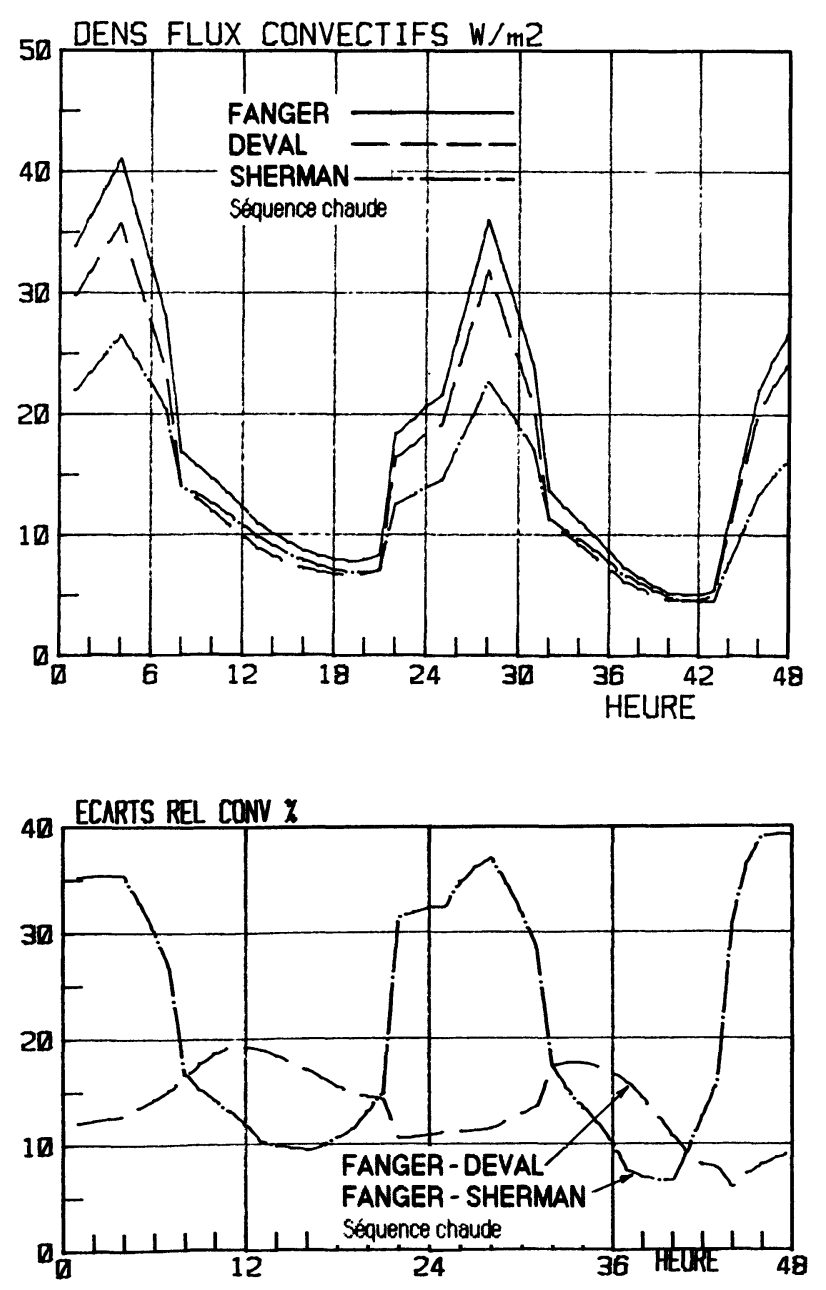

\subsection{ANAlyse détaillée Des ÉCARTS.}

5.4.1 Echanges convectifs. - Les résultats obtenus apparaissent à la figure 5 .

\section{* Ecarts Fanger-Sherman.}

Nous venons de voir que les modèles de Sherman et de Fanger étaient très proches en termes de réponse thermique globale $Y(X, t)$. On voit ici, par contre, que les écarts sur le mécanisme convectif peuvent atteindre $30 \%$. Ils seront encore plus élevés pendant la séquence chaude. Cela provient d'un choix de coefficients d'échanges convectifs différents induisant des écarts considérables. Ainsi, en convection naturelle, Sherman ne prend pas en compte le gradient de température qui s'établit entre la surface d'enveloppe du corps et l'air ambiant :

$$
\begin{aligned}
H_{\mathrm{C}, \mathrm{v}, 1} & =2,38\left|T_{\mathrm{v}, 1}-T_{1}\right|^{0,25} \\
h_{\mathrm{C}, 3} & =5,66(a-0,85)^{0,39} .
\end{aligned}
$$
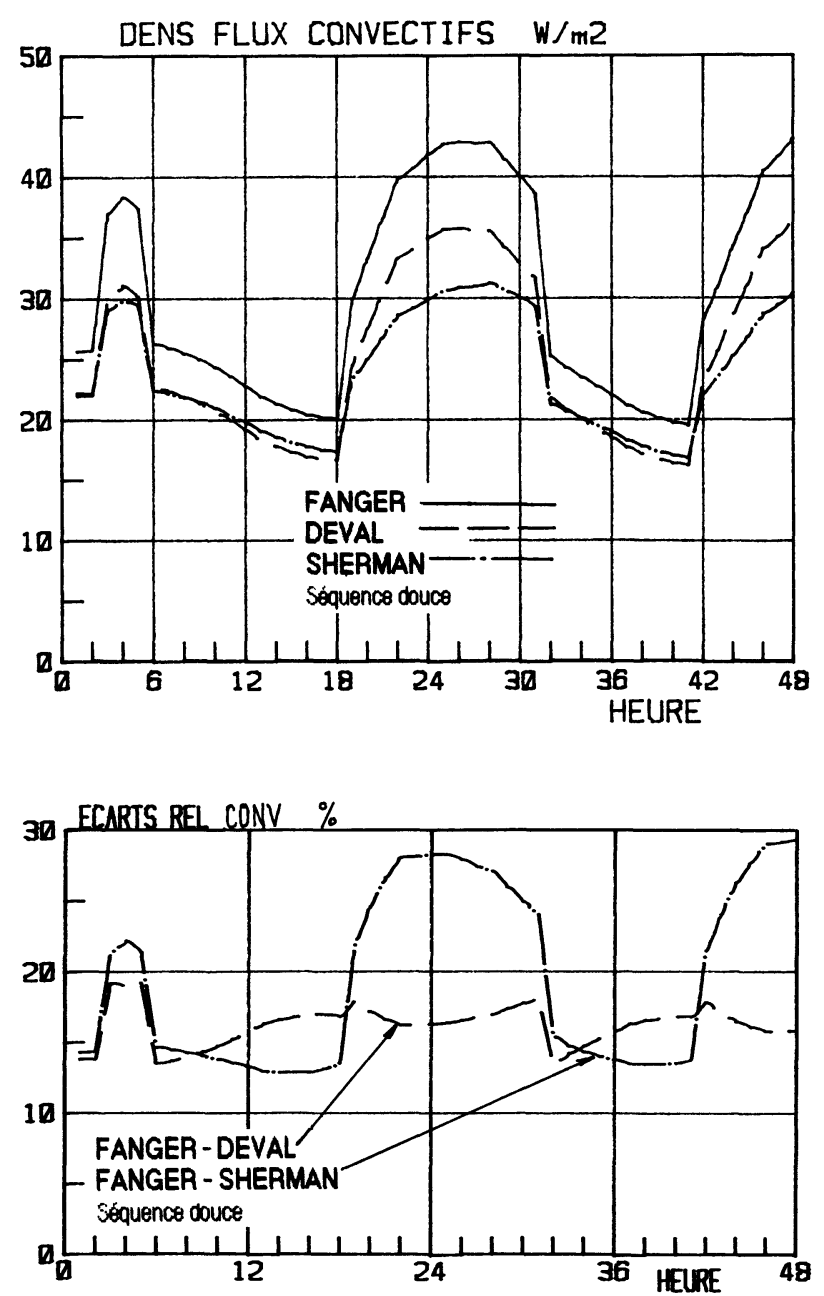

HEURE

Fig. 5. - Comparaison des modèles sur les échanges convectifs $E_{\mathrm{CV}}(X)$.

[Model comparison for convective heat exchange $E_{\mathrm{CV}}(X)$.] 
De même, en convection forcée, les expressions sont sensiblement différentes :

$$
h_{\mathrm{C}, \mathrm{v}, 1}=12,06 V_{\mathrm{a}}^{0,5} \quad h_{\mathrm{C}, 3}=8,3 V_{\mathrm{a}}^{0,53} .
$$

Pour les vitesses fréquemment rencontrées en ventilation d'été $\left(V_{\mathrm{a}}=0,25 \mathrm{~m} / \mathrm{s}\right)$, on obtient :

$$
h_{\mathrm{C}, \mathrm{v}, 1} \approx 6 \mathrm{~W} / \mathrm{m}^{2}{ }^{\circ} \mathrm{C} \quad h_{\mathrm{C}, 3} \approx 4 \mathrm{~W} / \mathrm{m}^{2}{ }^{\circ} \mathrm{C} \text {. }
$$

Soit un écart de $33 \%$, les échanges plus faibles de Sherman tendant à décaler son modèle vers des sensations plus chaudes.

* Ecarts Fanger-Deval.

La tendance est la même que dans le cas de Sherman, mais moins accentuée.

En situation de convection naturelle, le coefficient d'échange convectif est calculé de la même manière. Il est différent en convection forcée et plus faible de $17 \%$, ce qui est l'ordre de grandeur de l'écart de l'échange.

Une deuxième source d'écarts relève strictement du mode de calcul de la température de surface $T_{\mathrm{v}}$. La figure 6 montre, toutefois, que les valeurs de $T_{\mathrm{v}}$ obtenues ne divergent que dans le cas de la séquence douce (de l'ordre de $0,5^{\circ} \mathrm{C}$ ).

Enfin, la principale variante réside ici dans l'attribution de températures de surfaces différentes à la tête et aux mains. Cette variante n'affecte toutefois que $6 \%$ du total de l'échange convectif.

Comme dans le cas de Sherman, cette minoration des échanges, en séquence douce, tend à augmenter la valeur de la sensation $Y(X, t)$, ce que nous retrouvons à la figure 4 .

5.4.2 Echanges radiatifs. - Les résultats apparaissent à la figure 7 .

Par rapport aux échanges convectifs, on peut observer que la tendance s'inverse, c'est-à-dire que le modèle de Fanger réduit l'importance de ce mode d'échange. En valeur relative, ils demeurent importants, à certaines heures de la séquence. Ils sont en fait à peu près du même ordre en valeur relative.

Ces écarts sont en relation directe avec la formulation des échanges. L'expression de Fanger n'est pas linéarisée alors que c'est le cas de Sherman et de Deval.

Pendant la saison chaude, les différences entre les modèles s'estompent à certaines heures, mais demeurent importantes.

On peut, d'ores et déjà, remarquer que ces écarts inverses des échanges convectifs et radiatifs, tendent à se compenser pour rapprocher les valeurs des sensations de celles de la référence. Il est intéressant de noter que, si le résultat global sur $Y(X, t)$ ne diffère pas beaucoup, les valeurs distinguées des échanges varient considérablement. On peut donc conclure à une formalisation des transferts sensibles (conv. et ray.) assez divergente des 3 modèles.

5.4.3 Echanges secondaires (perspiration, conv. respiratoire, humid. respiratoire). - Ces 3 modes d'échanges, que nous avons décrits plus haut, ont été groupés sous le terme $E(X)$ :

$$
E(X)=E_{\mathrm{ps}}(X)+E_{\mathrm{cr}}(X)+E_{\mathrm{hr}}(X) .
$$

34

TEMP SURF VETURE $\quad C$

\section{FANGER}

DEVAL

32

Sequence chaude

30

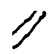

28

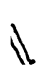

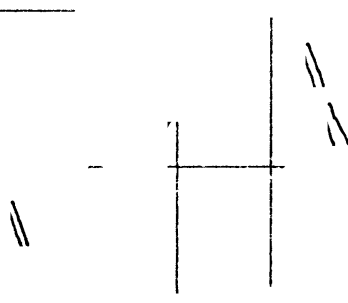

30

TEMP SURF VETURE ${ }^{\circ} \mathrm{C}$

28

Sequence douce

26

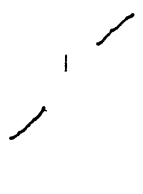

24

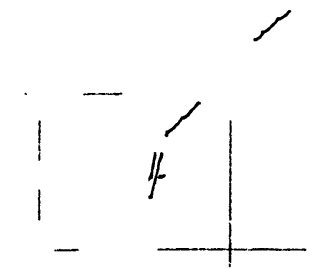
36 HEURE

Fig. 6. - Comparaison des modèles sur les températures d'enveloppe $T_{\mathrm{v}, X}$. Le modèle de Sherman n'est pas représenté puisque $T_{\mathrm{v}, 3}=T_{\mathrm{c}, 3}$ (cf. paragraphe 3.2).

[Model comparison for clothes temperature $T_{\mathrm{v}, X}$. The Sherman model is not showed because $T_{\mathrm{v}, 3}=T_{\mathrm{c}, 3}$ (cf. section 3.2).] 

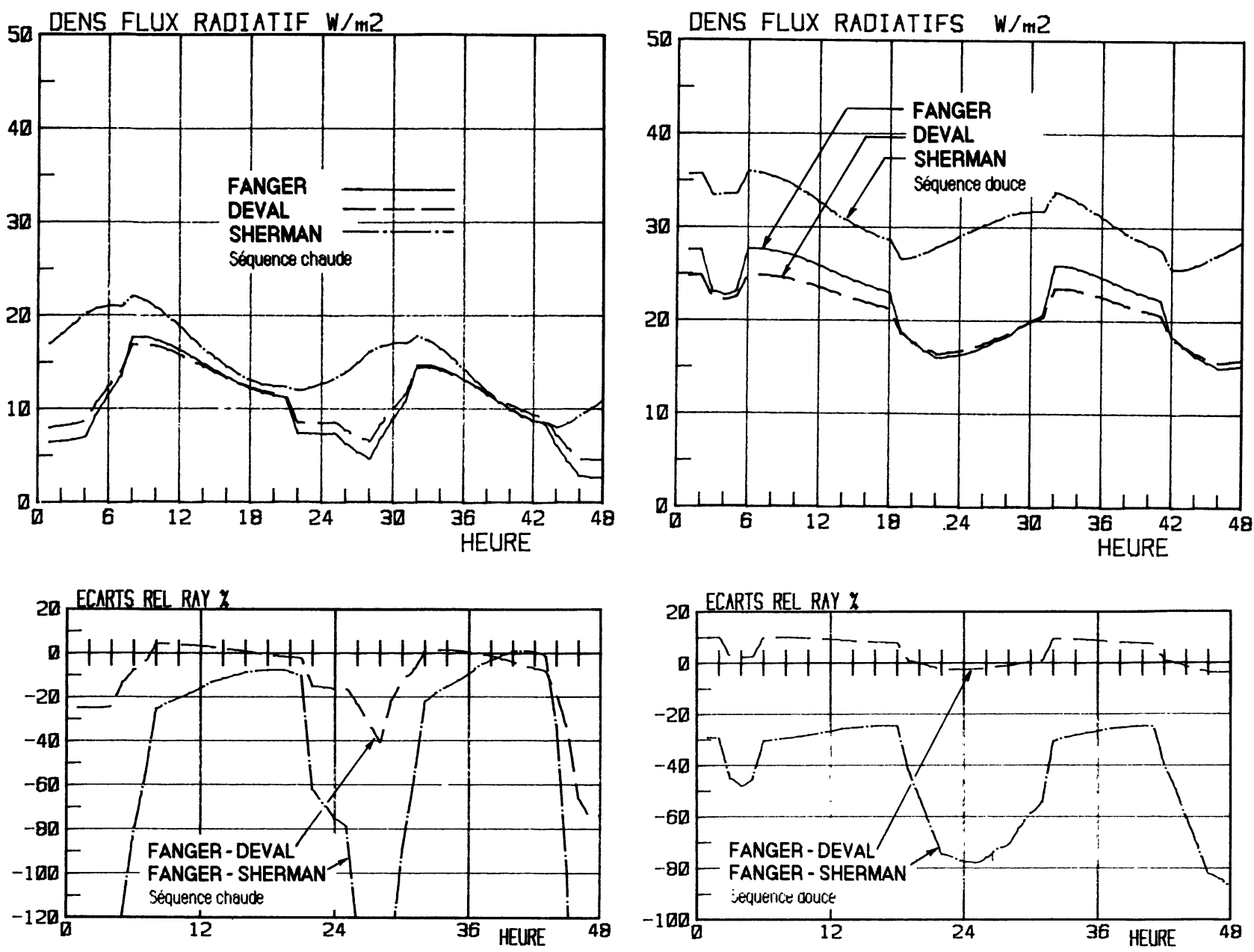

Fig. 7. - Comparaison des modèles sur les échanges radiatifs $E_{\mathrm{ry}}(X)$.

[Model comparison for radiative heat exchange $E_{\mathrm{ry}}(X)$.]

Les résultats sont présentés à la figure 8 .

On note, là encore, des différences remarquables entre les différents modèles. Elles sont, en valeur absolue, de l'ordre de 2 à $4 \mathrm{~W} / \mathrm{m}^{2}$. Elles proviennent aussi de formulations légèrement différentes.

\subsection{COMPARAISON DES RÉSULTATS SUR L'INDICE} DE QUALITÉ $q(X, t)$. - Si nous admettons que l'objet bâtiment peut se caractériser à l'aide de la fonction $q(X, t)$ et de ses grandeurs associées (moyenne, écart type), nous constatons que les modèles de Sherman et de Fanger conduisent à des résultats très proches dans le cas de la séquence douce. A l'inverse, le modèle de Deval diverge sensiblement et conduirait à des décisions constructives assez différentes.

Ces observations sont inversées dans le cas de la saison chaude où le modèle de Deval suit assez bien celui de Fanger. Le modèle de Sherman dérive en fin d'après-midi.

On remarque que l'analyse ramenée au plan de l'indice de qualité aggrave les différences entre les modèles. Or, en matière de caractérisation d'un espace et des composants dont résulte l'ambiance thermique, cet indice revêt une importance particulière. De tels résultats nous incitent à penser qu'il est nécessaire d'apporter des précisions complémentaires sur la modélisation des échanges thermiques, en définissant un modèle de référence bien recalé expérimentalement.

L'indice de qualité sera probablement, dans l'avenir, un critère de qualité permettant d'identifier la performance d'un ouvrage, en complément du coefficient de besoin énergétique. Les écarts auxquels nous aboutissons sur la fonction $q(X, t)$ nous laissent dubitatifs sur le choix du modèle à utiliser dans un système de $\mathrm{CAO}$ ou un système expert.

\section{Analyse de pertinence des modèles.}

Les 3 modèles que nous venons de décrire diffèrent peu quant à la manière de décomposer les échanges thermiques. Ils comportent par contre un certain nombre de variantes dans l'écriture des lois d'échanges. Ces variantes se caractérisent par : 

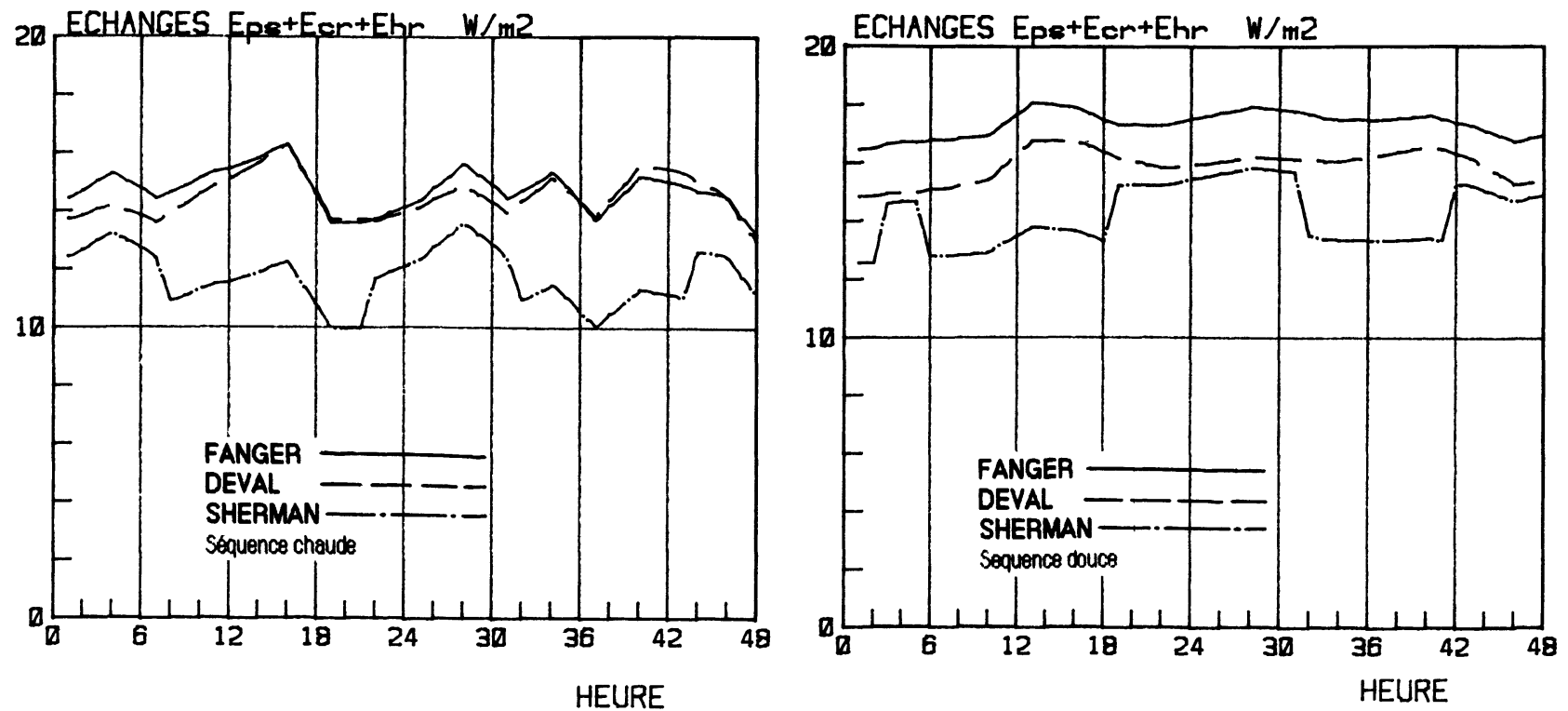

Fig. 8. - Comparaison des modèles sur les échanges secondaires: perspiration $E_{\mathrm{ps}}(X)$, conv. respiratoire $E_{\mathrm{cr}}(X)$ et humidité respiratoire $E_{\mathrm{hr}}(X)$.

[Model comparison for secondary heat exchange : evaporative heat loss from the skin $E_{\mathrm{ps}}(X)$, respiratory convective loss $E_{\mathrm{cr}}(X)$ and respiratory latent loss $E_{\mathrm{hr}}(X)$.]

- des simplifications de l'écriture des équations : linéarisation ou pas de ces équations, approximation de constance de certaines grandeurs...;

- des formulations différentes du phénomène, par le biais des coefficients ou des facteurs de proportionnalité.

Comme nous l'avons indiqué plus haut, il est délicat de trancher sur la meilleure qualité de formulation, car nous ne disposons que de peu d'expérimentations qui permettraient d'analyser séparément chaque mode d'échange. Mais la dispersion des résultats que nous venons d'observer et la sensibilité de ceux-ci au contexte thermique (séquence douce/séquence chaude) nous oblige à nous pencher sur la question générale de la modélisation des échanges corps-ambiance. Ne pouvant nous déterminer sur la validité intrinsèque de chaque modèle, nous aborderons la question de leur pertinence en relation avec leur utilisation dans les outils

6.1 LES TEMPS DE CALCUL. - Le temps de calcul est un élément important lorsque l'on cherche à implanter un modèle dans un projiciel de CAO. Toutefois, les algorithmes rencontrés ici sont d'une extrême simplicité. Les modèles de Fanger et de Deval nécessitent un calcul itératif lors de la recherche d'un zéro de fonction. Mais cette itération est, comme nous l'avons indiqué, ultra-rapide. Une petite expérimentation numérique montre, de fait, qu'un simple calcul en boucle, sans test, permet d'atteindre très finement la solution en 4 itérations (cf. travaux de Deval et Berger).

Quant au modèle de Sherman, il n'exige aucune itération. A titre d'exemple, nous obtenons l'ensemble des résultats recherchés, c'est-à-dire les grandeurs $Y(X, t)$ et $q(X, t)$, en $0,5 \mathrm{~s}$ sur un microordinateur pour une programmation basic.

En conclusion nous pouvons indiquer que, compte tenu de l'absence de difficulté algorithmique, le temps de calcul ne pose pas problème dans le cadre $\mathrm{du}$ choix du modèle et de son intégration dans un code de calcul plus général. La simplification apportée par Sherman, en termes de temps de calcul n'induit donc qu'un progrès très modeste.

\subsection{COHÉRENCE AVEC LA MODÉLISATION THERMI-} QUE DANS LE CONTEXTE CAO. - A l'heure actuelle, les logiciels disponibles pour simuler le comportement thermique d'un volume habitable se

On distingue principalement :

* Les codes dits « lourds », considérés comme les plus précis. Ils permettent de traiter avec une bonne précision, compatible avec l'analyse des critères caractérisant les enceintes, le comportement thermique de celles-ci. Ils exploitent des méthodes :

- numériques classiques (différences finies, éléments finis, modales) ;

- analytiques dans certains cas particuliers pour lesquels les solutions d'équations différentielles peuvent être approchées par l'outil mathématique. 
Ces codes de calcul sont principalement utilisés dans le contexte de la recherche, et leur usage n'est pas intensif. Ils ne sont exploités qu'exceptionnellement dans le cadre des pratiques professionnelles, dans le domaine du bâtiment par exemple.

* Les codes dits « légers », sont moins précis, mais faciles à implanter sur les calculateurs de petite capacité. Ils sont élaborés à partir des codes lourds, par simplification des algorithmes, en ménageant leur validité par rapport à l'expérimentation.

$\mathrm{Ce}$ sont ces codes rapides qui sont généralement intégrés aux outils de CAO [8].

Les modèles que nous venons de décrire sont plus adaptés à une utilisation chaînée avec les codes simplifiés, dans la mesure où les paramètres d'entrée leur sont communs. C'est, en particulier le cas de la température radiante moyenne $T_{\mathrm{r}}$, ou encore de la température d'air de l'ambiance, pour laquelle une hypothèse d'homogénéité est faite dans l'enceinte.

De ce point de vue, les 3 modèles sont cohérents avec les algorithmes de simulation thermique légers utilisés dans le domaine de la CAO.

Afin de compléter et d'expliciter notre propos, indiquons que d'autres modèles de description des échanges thermiques corps-ambiance sont actuellement développés. Les échanges radiatifs, par exemple, sont décrits avec plus de précision et le rapport géométrique entre le corps et l'enceinte, ainsi que l'hétérogénéité des températures de surface justifient l'utilisation, en amont, d'un code lourd d'analyse thermique de l'enceinte. A l'inverse, lorsque les contraintes de l'étude exigent l'exploitation d'un code lourd, il est plus cohérent de le chaîner avec un modèle plus sophistiqué.
6.3 CONSÉQuence SUR L'INDICE DE QUALITÉ $q(X, t)$. - La dispersion à laquelle nous aboutissons sur l'indice de qualité $q(X, t)$ rend délicat le choix du modèle (cf. Fig. 9). Cet indice, particulièrement important dans le contexte de CAO pour qualifier une enceinte pendant une séquence donnée, s'avère très sensible aux écarts observés sur les 3 modèles. On peut même noter qu'il tend à jouer le rôle d'amplificateur. Il serait donc utile, pour la manipulation de cet indice, de définir un modèle de référence unique permettant de comparer les solutions constructives des enceintes, au travers d'un indice moyen intégré sur la séquence :

$$
q=\frac{1}{n} \sum_{j=1}^{j=n} q(j \cdot \Delta t)
$$

\section{Conclusion.}

Les 3 modèles que nous avons testés présentent le même type d'approche des problèmes d'échanges thermiques entre le corps et l'enceinte dans laquelle évolue l'usager. Leur structure est équivalente, mais la formulation des flux présente quelques variantes. Ces variantes peuvent être considérées, en termes de lois fondamentales d'échanges, comme mineures, à la lecture des relations. Elles induisent toutefois des écarts considérables lors de l'évaluation des critères caractérisant les ambiances comme la sensation thermique ou l'indice de qualité.

Ces différences observées posent un double problème :

1) Celui du choix du modèle, pour les différentes applications dans le contexte industriel. La tendance
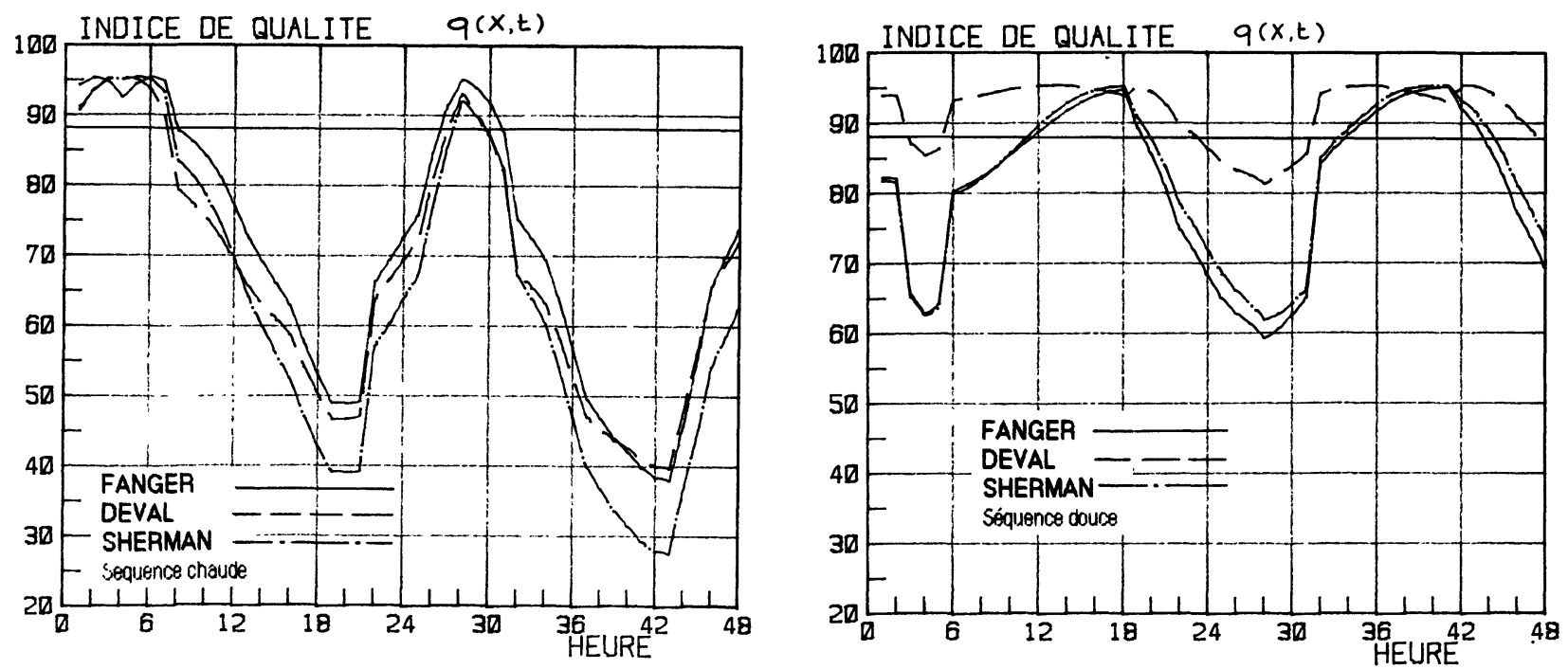

Fig. 9. - Comparaison des modèles sur l'indice de qualité $q(X, t)$.

[Model comparison for quality index $q(X, t)$.] 
logique poussera l'utilisateur à opter pour le modèle lui paraissant le plus adapté à son thème de travail, sans qu'il se rende compte qu'il introduit une dérive des résultats par rapport à une autre représentation. La difficulté surgit ensuite lors du rapprochement des indices, considérés comme critères de sélection de tel ou tel produit par des équipes de chercheurs ou d'ingénieurs différentes.

Il s'agit donc là du problème de la normalisation des outils employés par des partenaires ou des concurrents.

2) Celui de la qualité intrinsèque du modèle qui ne peut se repérer que par rapport à l'expérimentation. Nous avons, dans notre analyse, admis que le modèle de référence était celui du groupe Danois du Pr Fanger. Ce choix se justifie par la validation expérimentale que nous venons d'évoquer. Toutefois, celle-ci n'a été faite que pour des ambiances tempérées, du type de celles que nous rencontrons dans les bâtiments pendant la saison froide. Ainsi, la valeur de référence de ce modèle est-elle altérée pour les séquences chaudes auxquelles le corps sera soumis dans différents cas d'habitacles (véhicules, habitat, ...).

Il résulte de ces différentes considérations que la modélisation des échanges thermiques gagnerait à être approfondie à l'aide de représentations physi- ques plus sophistiquées. Ces travaux pourraient d'ailleurs donner lieu à une « rétro-réponse » vers la modélisation simplifiée en termes de recalage des modèles que nous venons d'analyser.

Malgré les défauts que nous venons de leur constater, on doit, aujourd'hui, reconnaître qu'ils demeurent des outils nécessaires dans certains domaines d'activité. Il suffit de prendre quelques précautions relatives à la cohérence des schémas de simulation pour les exploiter dans de bonnes conditions. Le chemin est toutefois ouvert vers une meilleure connaissance des systèmes thermiques, au sens de système physique, auxquels le corps humain se trouve associé.

\section{Annexe.}

Les coefficients $Y$ de Sherman utilisés dans le texte sont définis de la manière suivante :

$$
\begin{aligned}
Y_{\mathrm{ry}} & =F_{\mathrm{v}, 3} \cdot h_{\mathrm{r}, 3} \cdot T_{\mathrm{c}, 3} \cdot f(m) / m_{0} \\
Y_{\mathrm{CV}}= & {\left[0,0014 \cdot a+F_{\mathrm{v}, 3} h_{\mathrm{C}, 3} / m_{0}\right] \cdot T_{\mathrm{c}, 3} \cdot f(m) } \\
Y_{\mathrm{c}}= & {\left[0,0024 a+0,132 F_{\mathrm{p}, 3} h_{\mathrm{C}, 3} / m_{0}\right] \times } \\
\times T_{\mathrm{c}, 3} \cdot f(m) & \\
Y_{0}= & {[0,4+0,6 a]\left[f(m)-Y_{\mathrm{ry}}-Y_{\mathrm{CV}}-Y_{\mathrm{e}}\right] } \\
f(m)= & 1,6+17,6 \mathrm{e}^{-0,0362 m} .
\end{aligned}
$$

\section{Bibliographie}

[1] FANGer P. O., Thermal Comfort (MacGraw Hill) 1973.

[2] Deval J., Le confort en climat tempéré, Rev. Phys. Appl. 19 (1984) 513-531.

[3] Sherman M., A simplified model of thermal comfort, Energy and Buildings (Feb. 1985) p. 37-50.

[4] Depecker P., Brau J., Roux, Habitat climatique. Du calcul à la conception, Programme de recherche REXCOOP, Rapport final, Plan Construction, AFME (avril 1985).

[5] Vogh, Miller-Chagas, Confort thermohygrométrique, définition physiologique et détermination pratique des zones de confort thermique, Annales de l'ITBTP (juillet 1970).

[6] StolwiJk J. A. J., Mathematical model of thermoregulation. Physical and behavioral temperature regulation. J.A.O Hardy (Thomas Publisher, Springfield) Chap. 48.

[7] Cordier A., Thellier F., Modèle de bilan thermique du corps humain, Congrès Société Française des Thermiciens, Lyon (10 mars 1988).

[8] Roux J. J., Depecker P., Krauss G., Pertinence and performance of a thermal model adapted to a CAD context, International Congress PLEA, Porto (July 1988). 